\title{
Lufthaushalt in der Schweiz - einleitende Bemerkungen
}

Die regional ausgerichtete Atmosphärenforschung erlebte in den achtziger Jahren einen massiven Aufschwung, welcher auch in der Zukunft andauern dürfte. Die beiden Teildisziplinen mesoskalige Meteorologie und Klimatologie verdankten ihren Ausbau teilweise neuen methodischen Mitteln:

Größere Rechnerkapazitäten erlaubten zusammen mit den wachsenden Möglichkeiten des Einsatzes von Kleincomputern auch den kleineren Forschungsgruppen die Anwendung geeigneter numerischer Modelle. Parallel dazu konnten die physikalischen Modellierungstechniken im Windkanal oder in der Klimakammer ganz wesentlich verfeinert werden. Man denke etwa an neue Mikro-Meßmethoden oder an die Verbesserung der Simulation von dynamischen Prozessen über komplexer Topographie.

Ganz entscheidend wird die regionale Atmosphärenforschung zurzeit durch die wachsenden Umweltprobleme angeregt. Im Zentrum vieler Fragestellungen steht dabei die Luftverschmutzung, d.h. die Emission, die Ausbreitung und Umwandlung sowie die Immission und Deposition von Luftfremdstoffen.

Aus der Sicht der regionalen Meteorologie und Klimatologie interessieren vor allem jene Prozesse, welche diese Ausbreitung, d.h. den Transport und die turbulente Diffusion von festen, flüssigen oder gasförmigen Luftbestandteilen, bewerkstelligen. Vereinfachend bezeichnet man die Gesamtheit dieser Vorgänge sehr oft mit dem Sammelbegriff des Lufthaushaltes.

Ausgesprochen komplex gestalten sich diese Transportund Diffusionsprozesse über unebener Topographie. Die Atmosphäre wird durch Hügel und Gebirge sowohl dynamisch (mechanisch) als auch thermisch viel massiver modifiziert als über ebener Topographie. Oft erzeugen diese hügeligen Oberflächenstrukturen eigene Zirkulationssysteme oder Stagnationszonen, welche vom synoptischen Geschehen (z. B. ausgedrückt in Form des geostrophischen Windes) völlig abgekoppelt sind: Thermotopographische Windsysteme (Hang-, Berg- und Talwinde), stabile Kaltluftpools der Vorlandsenken mit Nebelmeeren usw.

In der Schweiz wurde derartigen Fragestellungen früh Beachtung geschenkt. Nicht zuletzt wegen der ausgezeichneten Meß-und Beobachtungsnetze im Alpenraum erhielt die Schweiz im Jahre 1981/82 Gelegenheit, das internationale meteorologische Gebirgsexperiment ALPEX zu beherbergen - ein Projekt, das den Forschern unseres Landes viele Impulse vermittelte. Kurz nach der ALPEX-Feldphase erfolgte der Start zum Nationalen Forschungsprogramm 14 "Lufthaushalt und Luftver- schmutzung in der Schweiz», welches kurze Zeit später durch das Zusatzprogramm "Waldschäden und Luftverschmutzung» aufgestockt wurde. Die Schlußpublikationen dieser Programme befinden sich im Druck, und bereits laufen die Arbeiten an einem neuen, interdisziplinären Projekt zum detaillierten Studium der raumzeitlichen Struktur des Sommersmogs über dem nördlichen Schweizer Alpenvorland (POLLUMET).

Das vorliegende Heft berichtet über Lufthaushaltsstudien, welche in verschiedenen Regionen unseres Landes von Geographen durchgeführt wurden. Im Vordergrund steht dabei die direkte und indirekte (via Nebel) Beschreibung von Strömungs- und Transportvorgängen. Dabei wird ein bewußter Ausgleich zwischen experimentellen und Modellstudien angestrebt.

Die erste Arbeit von Peter Schuhmacher befaßt sich mit den Resultaten einer ausgedehnten Windfelduntersuchung in Zürich. Mit Hilfe eines dichten Meßnetzes sowie von drei verschiedenen Computermodellen wurde nicht nur der mesoskalige Einfluß der Topographie abgeschätzt. An einem Beispiel wurde exemplarisch der mikroskalige Effekt des Schienenraumes beim Bahnhof studiert. Der zweite Beitrag von Beat Rickli, Heinz Wanner und Paul Filliger zeigt anhand eines Beispiels aus der Innerschweiz experimentelle und theoretische Möglichkeiten zur raumzeitlichen Charakterisierung des Ausbreitungsklimas über komplexer Topographie auf. Im dritten Aufsatz von Franz Xaver Troxler und Heinz Wanner wird der Nebel als Indikator benützt, um das Ausbreitungsklima über der Schweiz im Sinne der Mittelwertsklimatologie, aber auch der dynamischen Klimatologie oder Wetterlagenanalyse zu beschreiben. Jean-Michel Fallot untersucht im vierten Beitrag die thermotopographischen Windsysteme über der komplexen Topographie des Greyerzerlandes. Mit Hilfe des Vergleichs von numerischen und physikalischen (Laborsimulationen) Modellstudien mit experimentellen Resultaten weist er auf die komplexen Interaktionen zwischen dem synoptischen Antrieb und thermotopographischen Lokalströmungen hin. Urs Neu beschliesst die Serie der Arbeiten mit der Beschreibung eines einfachen, diagnostischen Windfeldmodells. Dieses erlaubt via Divergenzminimierung eine Interpolation des dreidimensionalen Windfeldes in Bodennähe und ist daher für die Berechnung von Rückwärts-Trajektorien verwendbar.

Heinz Wanner, Prof., Dr. phil. nat., Geograph. Institut der Universität Bern, Hallerstraße 12, 3012 Bern 